\title{
MR Imaging Characteristics of Myoepithelial Carcinoma Ex Pleomorphic Adenoma of the Palate: Rare Case Report
}

\author{
Byeong-Uk Jeon ${ }^{1}$, Ha Youn Kim ${ }^{1,}{ }^{*}$, In Kyu Yu ${ }^{1}$, Hyun-Jin Son ${ }^{2}$, Dong-Sik Chang ${ }^{3}$ and Young Do Jang ${ }^{4}$ \\ ${ }^{1}$ Department of Radiology, Eulji University Hospital, Daejeon, Republic of Korea \\ ${ }^{2}$ Department of Pathology, Eulji University Hospital, Daejeon, Republic of Korea \\ ${ }^{3}$ Department of Otorhinolaryngology-Head and Neck Surgery, Eulji University Hospital, Daejeon, Republic of Korea \\ ${ }^{4}$ Department of Otorhinolaryngology-Head and Neck Surgery, Bon ENT Clinic, Daejeon, Korea \\ "Corresponding author: Department of Radiology, Eulji University Hospital, 95, Dunsanseo-ro, Seo-gu, Daejeon, Republic of Korea Republic of Korea. Tel: +82-1085282735, Email: \\ mayon2@naver.com
}

Received 2019 February 21; Revised 2019 August 26; Accepted 2019 September 04.

\begin{abstract}
Carcinoma ex pleomorphic adenoma is an uncommon tumor of salivary gland; moreover, myoepithelial carcinoma of palate is exceedingly rare. This report documents a case of carcinoma ex pleomorphic adenoma arising in the hard palate. A 51-year-old woman presented with foreign body sensation without pain. Preoperative biopsy was performed and resulted in pleomorphic adenoma of the hard palate. MR imaging findings were well circumscribed, dome-shaped, lobulated mass with hyposignal intensity at peripheral fibrous capsule on T2WI. The mass showed internal cyst, hemorrhage, necrosis and heterogeneous enhancement. Because it showed both benign and malignant findings, the radiologic diagnosis was likely to be a malignant tumor of palate. Postoperative pathological analysis revealed invasive myoepithelial carcinoma ex pleomorphic adenoma.
\end{abstract}

Keywords: Myoepithelial Carcinoma Ex Pleomorphic Adenoma, Myoepithelial Carcinoma, Conventional Magnetic Resonance Image, Palatal Tumor

\section{Introduction}

Myoepithelial carcinoma (MC) is a very rare tumor, found in only about $1.5 \%$ of salivary gland tumors. MC was originally described by Donath et al. as a carcinoma in 1972, made by duct epithelial cells and myoepithelial cells (1). MC is usually generated from the tissues of the head and neck, and in most cases occurs in salivary glands and parotid glands (2). MC in the palate is especially rare, and only 28 cases have been reported in the literature (3). Myoepithelial carcinoma ex pleomorphic adenoma (MCXPA), defined as MC with malignant transformation in a pleomorphic adenoma, is extremely rare. Recently, there have been several reports of CT or MRI findings for tumors of the palate $(4,5)$. However, so far no reports have been made regarding the MRI features of the MCXPA arising at the palate. Here, we present a case for MCXPA that occurred at the palate and review the MRI findings of the tumor.

\section{Case Presentation}

A 51-year-old female patient came to our institution in March 2018 with a one-year history of a growing mass caus- ing discomfort inside her oral cavity. A soft mass, approximately $30 \times 30 \mathrm{~mm}$, was observed on the right side of the hard palate, without tenderness during physical examination. Initial pathological diagnosis by biopsy suggested benign salivary gland tumor including pleomorphic adenoma (PA). Informed consent was obtained before all diagnostic tests were carried out.

The patient had undergone magnetic resonance (MR) scans just after outpatient medical treatment, and the appearance of the tumor was about $2.6 \times 2.2 \times 3.0 \mathrm{~cm}$-sized. The tumor was a well-circumscribed, dome-shaped, lobulated mass with an inner multiseptated, hemorrhagic, cystic portion (fluid-hemorrhage level was shown in each septate cyst) and a peripherally located fibrous capsule with hyposignal intense on T1- and T2-weighted images in the right posterior hard palate. Post-contrast enhanced images showed a heterogeneously enhanced lobulated mass with an inner necrotic portion and non-enhanced fibrous capsule (Figure 1). However, adjacent bone invasion, perineural extension, and sinonasal involvement were not observed. We were able to make a differential diagnosis of malignant tumor originating from the minor sali- 
vary gland, such as myoepithelial carcinoma ex pleomorphic adenoma (MCXPA), acinic cell carcinoma or polymorphous low-grade adenocarcinoma or benign minor salivary gland tumor, such as pleomorphic adenoma or myoepithelioma.

Tumor resection was performed with sufficiently safe margins. The tumor extended over the hard palate and soft palate, and was approximately $3.3 \times 3.0 \times 1.8 \mathrm{~cm}$. By microscopic examination, the size of the tumor was measured at a maximum diameter of $2.5 \mathrm{~cm}$, and was defined as PT2 due to lack of extraparenchymal extension. There were no lymph node metastases, and the tumor was classified as histologic grade 2 , showing a moderate differentiation. The tumor encapsulated with fibrotic capsule with internal cavitary lesion. A foci of remnant pleomorphic adenoma was seen, and tumor cells suspected of invading through a capsule in the focal area were observed. The tumor was mainly composed of a solid sheet of plasmacytoid cells, which had moderate nuclear pleomorphism with frequent mitoses (with up to 12 per high-power field) and abundant eosinophilic cytoplasm (Figure 2). Additionally, by evaluation by immunostaining, tumor cells were positive for cytokeratin-7 (CK7), S-100, p63, p53, and Cerb2, but smooth muscle actin (SMA) was negative in the tumor cells. The nuclei stained positive for mindbomb E3 ubiquitin protein ligase 1(MIB-1) moderately (Ki-67 labeling index is about 10\%). These findings support the development of MCXPA.

Two weeks later, the patient went through follow up fluorodeoxyglucose positron emission tomography computed tomography (FDG-PET/CT). The scan showed no metastases and abnormal lymphadenopathy. There was an increase in FDG uptake around the site of the previous tumor, but it was considered a postoperative lesion. In addition, no new lesions were observed in the laryngoscope, which was conducted after two months, and the patient is now under follow-up observation.

\section{Discussion}

Myoepithelial carcinoma (MC), also known as malignant myoepithelioma, is an atypical malignant neoplasm in the salivary glands, and accounts for $1.5 \%$ of all salivary gland tumors (1). It was first reported by Donath in 1975, and was classified as a type of salivary tumor by the World Health Organization in 1991, and the definition was updated in 2005 (2). The invasiveness of MC can vary from regionally assertive to a highly metastatic tumor. Malignant myoepitheliomas arise as a malignant transforma- tion in the setting of a benign pleomorphic adenoma or a benign myoepithelioma. Other malignant myoepitheliomas arise de novo (2). Pathogenic outbreaks of the tumor are not well known, and almost 7\% of the cases arise from the palatal minor salivary glands $(6,7)$. Symptoms of MC are similar to those of other salivary gland tumors: a bulging mass with or without pain, facial palsy, and fixation of the mass to the underlying structures (8). In this case, the patient had foreign body sensation caused by the mass, without any other symptoms.

MRI and CT are useful for describing characteristics of palate tumors, complete extension of the tumor, and identifying any localized lymphadenopathy. On CT and MR imaging, benign palatal tumors have smooth or lobulated margins, homogeneous to various degrees of enhancement (4). Pleomoprhic adenoma shows characteristic fibrous capsule appearing as hypointense on T2-weighted images (4). Low-grade malignant tumors have a generally benign appearance, but microcysts or irregular cystic spaces, hemorrhagic or necrosis are occasionally observed on CT and MR images. High-grade malignant tumors show poorly delated margin, invasion to adjacent structure (nasal cavity, maxillary sinuses) and perineural spread (5). In MR imaging of the MCXPA presented here, the lesion appeared as a lobulated, heterogeneous enhancing mass with internal fluid-hemorrhage level and a necrotic portion, suggesting a malignant tumor. It accompanied a peripheral hypointense fibrous capsule on T2-weighted images, suggesting a characteristic feature of benign condition, especially pleomorphic adenoma. We were able to make a differential diagnosis of malignant tumor originating from the minor salivary gland, such as MCXPA, acinic cell carcinoma or polymorphous low-grade adenocarcinoma or benign minor salivary gland tumor, such as pleomorphic adenoma or myoepithelioma.

Some reports describe the relationship between the immunohistochemical (IHC) results and the clinical process. The overexpression of the Ki67 stain reflecting cell proliferation may be a useful indicator of poor prognosis in MC. Nagao et al. reported that four out of five patients representing overexpression of nuclear Ki67 (MIB1-index $>30 \%$ ) died of the disease (9). In our case, the prognosis is expected to be good by this metric (MIB1-index approximately 10\%).

So far, no consensus has been reached regarding the treatment of head and neck MC because of its rarity. The first choice of local tumors is extensive surgical excision. Although the effectiveness of radiotherapy is unclear, additional radiotherapy with or without chemotherapy is rec- 

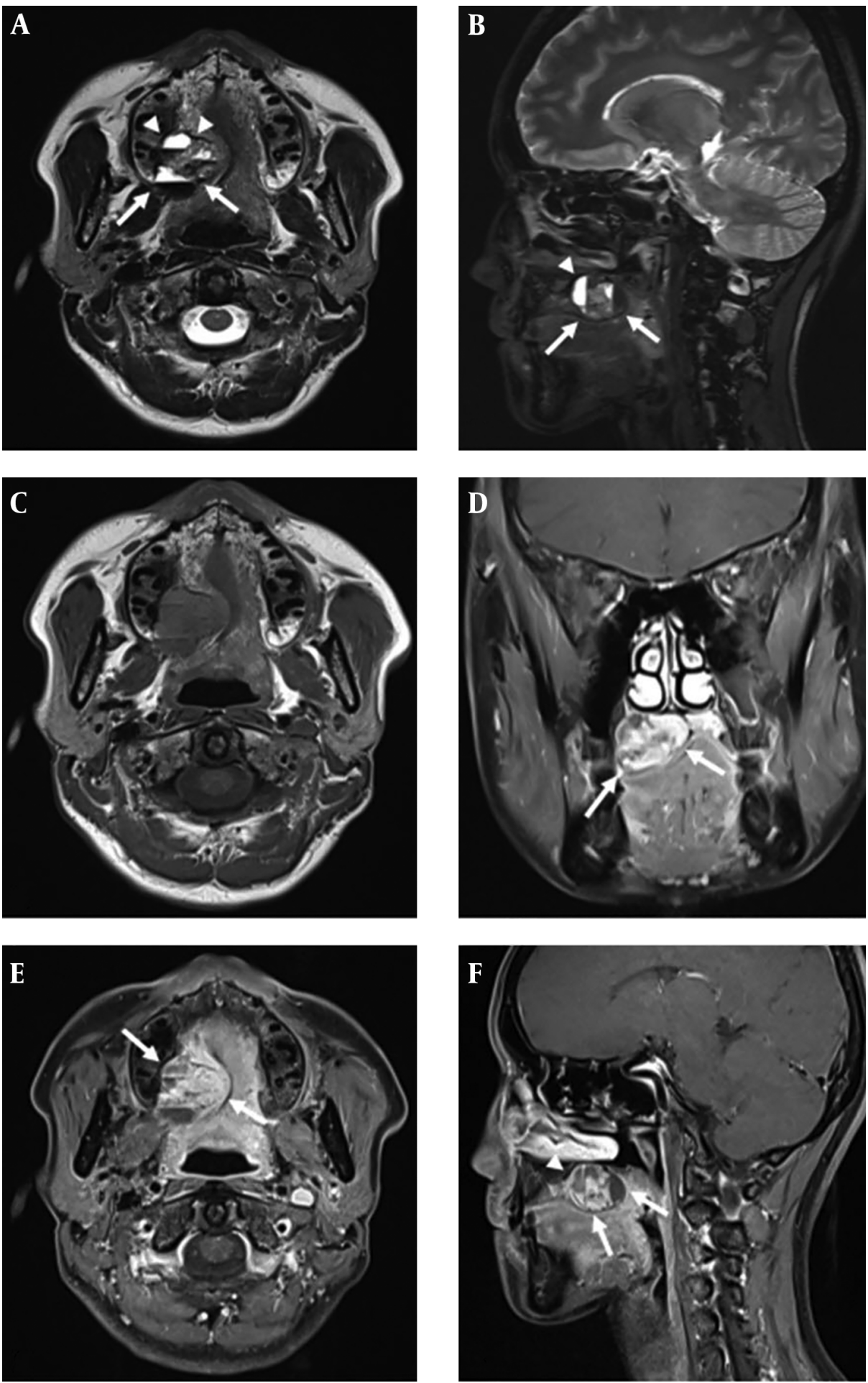

Figure 1. MRI study of a 51-year-old female patient with one-year history of a growing soft mass in the right hard palate. Axial(A) and sagittal(B) T2WI show well defined, dome shaped, lobulated mass (arrow) in the right posterior hard palate with peripherally hypointensed fibrous capsule (arrow head) and inner multiple septated hemorrhagic cyst portion (fluid-hemorrhage level was detected in each septated cyst). Axial TiWI shows intact cortical bone separating the mass (C). Coronal (D), axial (E) and sagittal (F) post-contrast enhanced TiWI show heterogeneously enhanced lobulated mass (arrow) with inner necrotic portion and non-enhanced fibrous capsule (arrow head). 

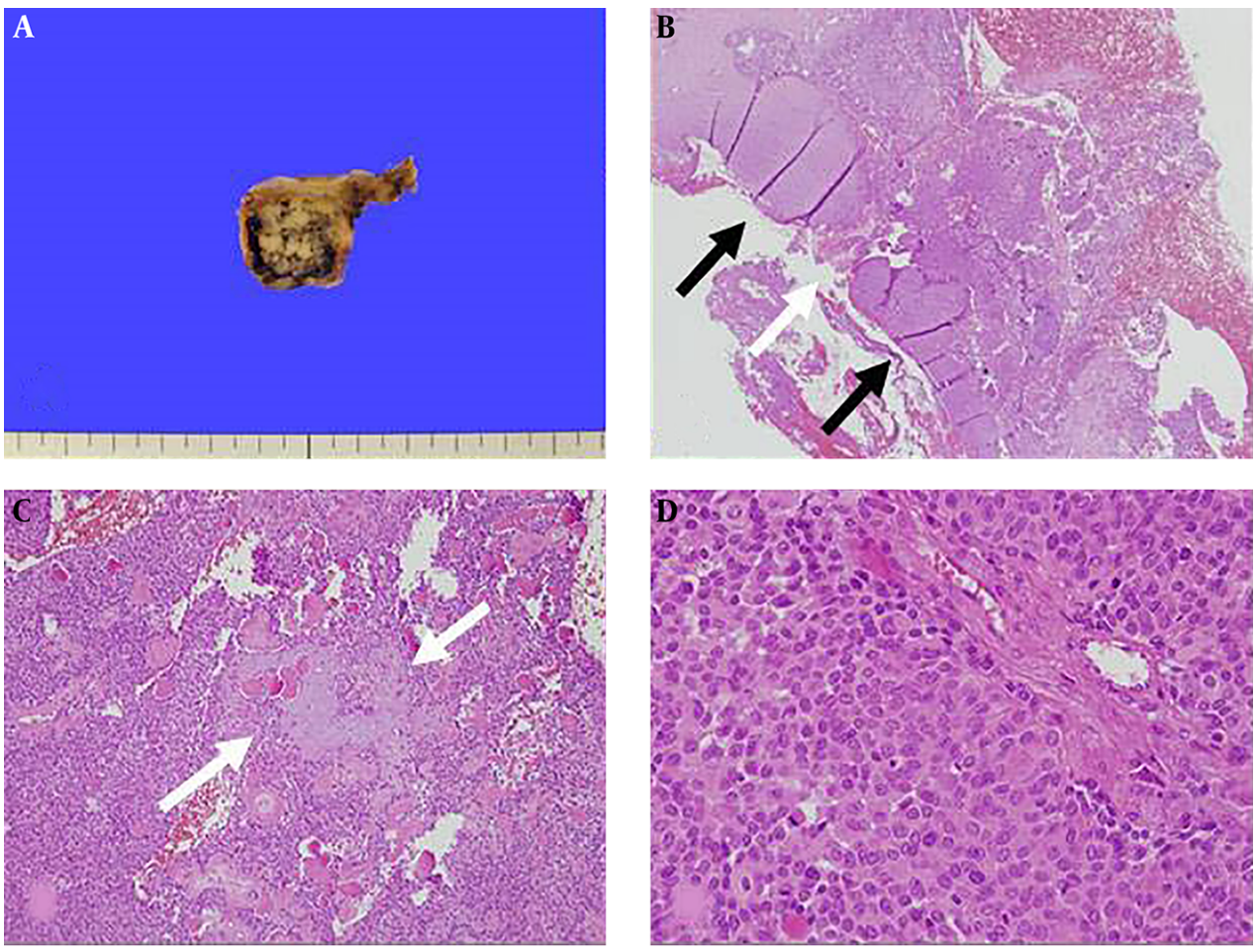

Figure 2. A, The tumor extended over the hard palate and soft palate, and was approximately $3.3 \times 3.0 \times 1.8 \mathrm{~cm}$ in dimensions. It encapsulated and had internal cavitary lesion. B, Fibrotic capsule (black arrows) was seen and suspicious tumor cells invaded through a capsule in the focal area (white arrow). (Hematoxylin and Eosin [H\&E] stained $\times 40$ ). C, A foci of remnant pleomorphic adenoma portion as seen (white arrows). (H\&E stain, $\times 100) \mathrm{D}$, Frequent mitoses was seen at the tumor $(\mathrm{H} \& \mathrm{E}$ stain, $\times 400$ ).

ommended for advanced malignant tumors with residual tumor, perineural, lymphatic or vascular invasion (10). In most cases, chemotherapy is administered as either adjuvant therapy, or alone for distant metastasis (11). MC originating from the minor salivary gland have been reported to have a relatively good prognosis (12). However, it is controversial whether the prognosis of MCXPA is more positive than de novo MC.

We present a rare case of myoepithelial carcinoma ex pleomorphic adenoma of the palate. An understanding of the MRI features helps radiologists to make correct diagnoses, and also directs physicians to the appropriate clinical diagnoses and treatment.

\section{Footnotes}

Authors' Contributions: Study concept and design: Ha Youn Kim; analysis and interpretation of data: Byeong-Uk Jeon, Dong-Sik Chang and Hyun-Jin Son; drafting of the manuscript: Byeong-Uk Jeon, Ha Youn Kim, and Hyun-Jin Son; critical revision of the manuscript for important intellectual content: In Kyu Yu and Young Do Jang; supervision:
Ha Youn Kim

Conflict of Interests: The authors declare that they had no conflict of interests.

Ethical Approval: This case report was approved by the Institutional Ethical Review Board and the patient's informed consent was waived (EMC 2019-03-032-001).

Financial Disclosure: None declared.

Funding/Support: No funding/support was received for this study.

\section{References}

1. Donath K, Seifert G, Schmitz R. [Diagnosis and ultrastructure of the tubular carcinoma of salivary gland ducts. Epithelial-myoepithelial carcinoma of the intercalated ducts]. Virchows Arch A Pathol Pathol Anat. 1972;356(1):16-31. German. [PubMed: 4340536].

2. Barnes L, Eveson JW, Sidransky D, Reichart P. Pathology and genetics of head and neck tumours. 9. IARC; 2005.

3. Thayumanavan B, Vani NV. Myoepithelial carcinoma of palate: Case report. Indian J Pathol Microbiol. 2014;57(1):85-8. doi: 10.4103/03774929.130906. [PubMed: 24739839].

4. Kato H, Kanematsu M, Makita H, Kato K, Hatakeyama D, Shibata T, et al. CT and MR imaging findings of palatal tumors. Eur J 
Radiol. 2014;83(3):e137-46. doi: 10.1016/j.ejrad.2013.11.028. [PubMed: 24377674].

5. Zheng Y, Xiao Z, Zhang H, She D, Lin X, Lin Y, et al. Differentiation between benign and malignant palatal tumors using conventional MRI: A retrospective analysis of 130 cases. Oral Surg Oral Med Oral Pathol Oral Radiol. 2018;125(4):343-50. doi: 10.1016/j.oooo.2018.01.006. [PubMed: 29477604].

6. Olsen KD, Lewis JE. Carcinoma ex pleomorphic adenoma: A clinicopathologic review. Head Neck. 2001;23(9):705-12. doi: 10.1002/hed.1100. [PubMed: 11505478].

7. Furukawa M, Suzuki H, Matsuura K, Takahashi E, Suzuki H, Tezuka F. Carcinoma ex pleomorphic adenoma of the palatal minor salivary gland with extension into the nasopharynx. Auris Nasus Larynx. 2001;28(3):279-81. doi: 10.1016/s0385-8146(01)00052-9. [PubMed: 11489377].

8. Kane SV, Bagwan IN. Myoepithelial carcinoma of the salivary glands: A clinicopathologic study of 51 cases in a tertiary cancer center. Arch Otolaryngol Head Neck Surg. 2010;136(7):702-12. doi: 10.1001/ar- choto.2010.104. [PubMed: 20644067].

9. Nagao T, Sugano I, Ishida Y, Tajima Y, Matsuzaki O, Konno A, et al. Salivary gland malignant myoepithelioma: a clinicopathologic and immunohistochemical study of ten cases. Cancer. 1998;83(7):1292-9. doi: 10.1002/(sici)1097-0142(19981001)83:7<1292::aid-cncr4>3.0.co;2-1. [PubMed: 9762928].

10. Khalesi S. Retraction: A review of Carcinoma ex Pleomorphic Adenoma of the salivary glands. World J Pathol. 2017;6(6).

11. Wakasaki T, Kubota M, Nakashima Y, Tomonobe E, Mihara T, Fukushima J. Invasive myoepithelial carcinoma ex pleomorphic adenoma of the major salivary gland: Two case reports. BMC Cancer. 2016;16(1):827. doi: 10.1186/s12885-016-2871-3. [PubMed: 27793123]. [PubMed Central: PMC5084397].

12. Yang S, Zeng M, Zhang J, Chen X. Clear cell myoepithelial carcinoma of minor salivary gland: A case report. Int J Oral Maxillofac Surg. 2010;39(3):297-300. doi: 10.1016/j.ijom.2009.10.013. [PubMed: 19939628]. 